\title{
Trimetallic ruthenium complexes based on 1,3,5-tris(4-ethynylbenzonitrile)benzene
}

\author{
Zsolt Csók
}

Received: 23 March 2009/Accepted: 6 May 2009

(C) Springer Science+Business Media B.V. 2009

This article has been withdrawn due to disputed authorship.

This article has been withdrawn due to disputed authorship.

Z. Csók ( $\bigotimes)$

Centro de Química da Madeira, Departamento de Química da

Universidade da Madeira, Campus da Penteada, 9000-390

Funchal, Portugal

e-mail: zscsok@gmail.com 